\title{
Corrigendum: A doublecortin containing microtubule-associated protein is implicated in mechanotransduction in Drosophila sensory cilia
}

\author{
S. Bechstedt, J.T. Albert, D.P. Kreil, T. Müller-Reichert, M.C. Göpfert \& J. Howard
}

Nature Communications 1:11 doi: 10.1038/ncomms1007 (2010); Published 12 Apr 2010; Updated 13 May 2014

After reanalysing the original electron micrographs, as well as new ones, we discovered that, contrary to what was stated in the paper, the ciliary dilations in DCX-EMAP mutants do contain electron-dense material (arrow in new Fig. 7b). Furthermore, what we interpreted as a ciliary dilation without electron-dense material (Fig. 5d,e in the original Article) was a different structure, namely a swelling of the cilium. The swelling is distinguished from the dilation because it is located approximately half way between the dilation and the basal body (arrow in Fig. 7e). The swelling contains no electron-dense material. Swellings were also observed in wild-type cells (arrows in Fig. 7g).

Our misidentification of the swelling as a ciliary dilation does not contradict our hypothesis that the ciliary dilation may be the site of mechanotransduction in Johnson's organ. Rather, it weakens the argument by taking away one of the two lines of evidence in favour of the hypothesis. The other argument, that the essential mechanotransduction protein DCX-EMAP localizes to the ciliary dilation region, remains valid.

We thank Xin Liang for identifying this error and Monalisa Mishra, who helped with the follow-up electron microscopy. 
f02655
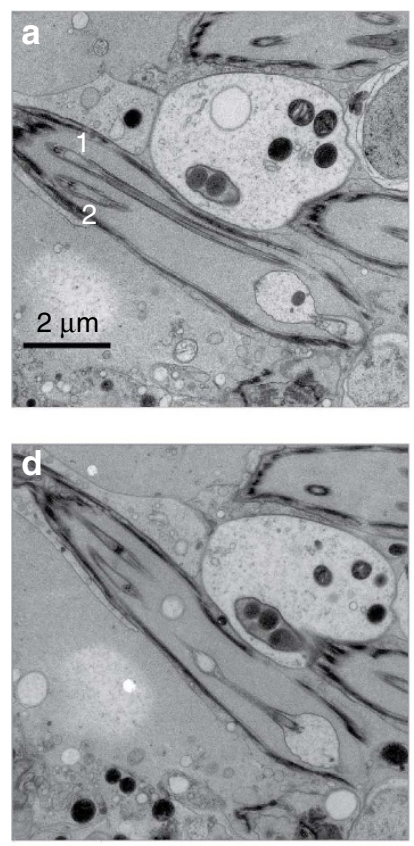

wild type

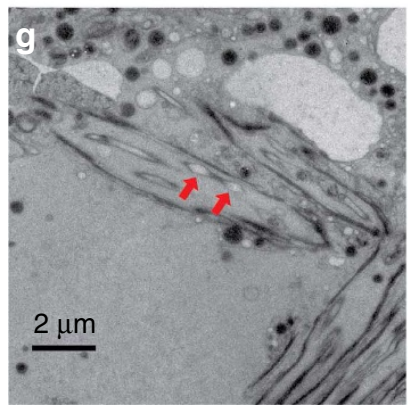

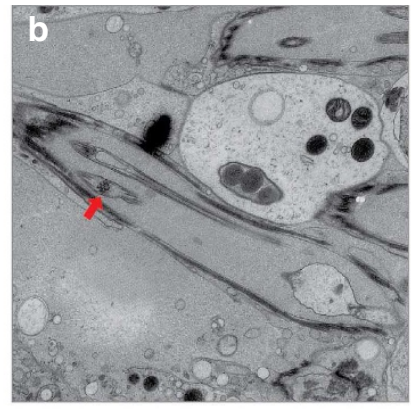
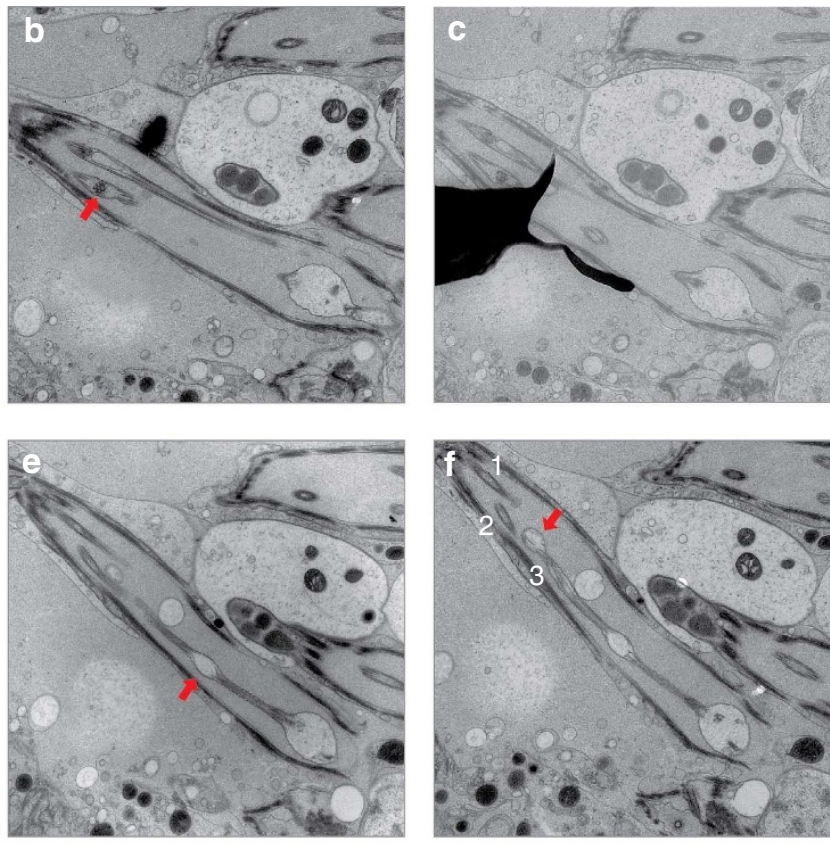

Figure 7 | Scolopale mechanoreceptors in the DCX-EMAP f02655 mutant have dilations with electron-dense material. (a-f) Serial sections of a scolopidium in the f02655 mutant. (a) Two of the three sensory cells are marked 1 and 2. (b) The dilation in cell 2 contains electron-dense material (arrow). (c) An intermediate section. (d) Another intermediate section. (e) A swelling is indicated by an arrow. Fig. 5d,e in the original Article comes from this section. (f) The three cells in the scollapidium are marked 1, 2 and 3. The swelling in e is located in cell 3. Note that cell 3 has an empty dilation (arrow). (g) Two swellings in a wild-type cell (arrows) showing that the swellings are not contingent on the f02655 mutation. 